\title{
Prevención y resolución del conflicto en educación física desde la perspectiva de los adultos miembros de la comunidad educativa en las comunidades de aprendizaje Prevention and conflict resolution in Physical Education from the perspective of the adult members of the educational community in learning communities
}

\author{
Sara Figueras Comas*, Jordi Calvo Lajusticia**, Marta Capllonch Bujosa* \\ *Universitat Ramon Llull. *Universitat de Barcelona.
}

\begin{abstract}
Resumen: El presente artículo está vinculado a un proyecto centrado en la superación de conflictos en educación física mediante el modelo comunitario. La parte del estudio a la que se refiere este artículo tiene por objetivo analizar las opiniones, creencias y valoraciones que los miembros adultos (profesorado, familiares y voluntariado) de las Comunidades de Aprendizaje realizan sobre las causas de los conflictos que se producen en la educación física y las formas de abordarlos.La investigación se ha desarrollado bajo el prisma de la metodología comunicativa crítica; y la recogida de información se ha llevado a cabo en 6 comunidades de aprendizaje ( 3 en Cataluña y 3 en el País Vasco). La participación en este estudio de los miembros adultos de las comunidades se concretó en la realización de entrevistas en profundidad (6 profesores, 6 familiares, 6 voluntarios); a partir de las cuales se realizó el correspondiente análisis cualitativo/comunicativo.Los resultados obtenidos y las conclusiones que se derivan de los mismos se organizan en base a dos aspectos principales: la identificación de las barreras que dificultan la resolución comunitaria de los conflictos (relacionadas con las posibilidades de éxito, la influencia de los medios de comunicación, la prevalencia de otros modelos, las dificultades para la comunicación y la normalización del conflicto); la identificación y propuesta de estrategias que favorecen la resolución comunitaria de los conflictos (relacionadas con el consenso de las normas, la orientación de la asignatura y la gestión de la competición).
\end{abstract}

Palabras clave: educación física, prevención del conflicto y resolución del conflicto, comunidades de aprendizaje, comunidad educativa.

\begin{abstract}
This article is linked to a project focused on overcoming conflicts in physical education through community model. The part of the study referred to in this article aims to analyse the opinions, beliefs and assessments that adult members (teachers, family and volunteers) of Learning Communities expressed on the causes of the conflicts in physical education and the ways to address them. The research has been developed under the perspective of critical communicative methodology; and data collection was carried out in 6 learning communities (3 in Catalonia and 3 in the Basque Country). Adult community members participated by responding in-depth interviews ( 6 teachers, 6 relatives, 6 volunteers); from which the qualitative / communicative analysis was carried on.The results and the conclusions are organized according to two main aspects: identification of barriers to community conflict resolution (related to chances of success, influence of the media, prevalence of other models, difficulties in communication and acceptance of conflict as normal); identification and suggestion of strategies that favour community conflict resolution (related to rules agreement, subject aiming and competition management).
\end{abstract}

Key words: physical education, conflict prevention and conflict resolution, learning communities, educational community.

\section{Introducción}

Este artículo presenta parte de los resultados obtenidos en la investigación realizada a través del proyecto financiado por el Ministerio de Educación y Ciencia en el marco del Plan Nacional I+D+i, con título: Juega, dialoga y resuelve (2008-2011): la superación de conflictos en educación física mediante el modelo comunitario. Diseño de un programa específico para Comunidades deAprendizaje (referencia: SEJ200761757/EDUC). En el proyecto se proponía el estudio de las vías para la resolución de conflictos que se producen en la educación física escolar en las Comunidades de Aprendizaje. El objetivo general se centraba en identificar los conflictos más comunes que se producen en los centros escolares y en las clases de educación física, así como, potenciar los mecanismos necesarios para su superación mediante la educación física, generando actitudes positivas e inclusivas extrapolables a otros ámbitos.

El incremento de los problemas de convivencia y del conflicto en las escuelas en los últimos años ha generado una evidente preocupación en las comunidades educativas y en toda la sociedad en general. En los artículos anteriores del presente monográfico se ha abordado la naturaleza y los distintos orígenes del conflicto, así como, sus formas de intervención y tratamiento en las escuelas. El presente artículo pretende focalizar el discurso en la percepción del conflicto que tienen los miembros adultos de la comunidad educativa, en las escuelas que son comunidades de aprendizaje.

Fecha recepción: 05-12-13- Fecha envío revisores: 05-12-13- Fecha de aceptación: 10-12-13 Marta Capllonch Bujosa

Campus Mundet, Edifici Llevant, 3a planta, despatx 317,

Passeig del Vall d'Hebron 171, 08035 Barcelona,

mcapllonch@ub.edu
La necesidad de abordar el conflicto desde la perspectiva del conjunto de miembros adultos de la comunidad educativa, y no reducirlo únicamente al colectivo de los profesores/as responde a dos premisas importantes que deben ser argumentadas. La primera premisa se refiere al reconocimiento actual y generalizado de la importancia que tiene la participación de los familiares y otros miembros de la comunidad en los centros escolares. En este sentido, recientes investigaciones asocian este tipo de participación a un mejor rendimiento académico (García Yeste, Leena \& Petreñas, 2013), así como, a una mayor motivación de los alumnos, que influye finalmente en el comportamiento y competencia social de los niños y niñas (Gázquez, Pérez \& Carrión, 2011). Así se recoge la necesidad que miembros adultos, participen de la dinámica de la escuela como propuesta de éxito escolar entre los alumnos y alumnas.

La segunda premisa que clarifica la importancia de abordar el conflicto desde la perspectiva global de los miembros adultos de la comunidad va vinculada a la propia naturaleza de las Comunidades de Aprendizaje y a sus relaciones con el entorno. Las Comunidades de Aprendizaje representan una experiencia de transformación social y cultural en las escuelas, que tienen por objetivo proporcionar una educación de calidad para todas las personas como respuesta a los retos que plantea la sociedad de la información (Elboj, Puigdellívol, Soler \& Valls, 2002). Este proceso de transformación se fundamenta en la participación de la comunidad, barrio o pueblo, en todos los espacios y procesos escolares. El papel de la comunidad en las Comunidades de Aprendizaje es vital, ya que la educación de éxito se entiende como una responsabilidad compartida entre el centro escolar y todo el entorno del alumnado a través del aprendizaje dialógico (Aubert, Flecha, García Yeste, Flecha \& Racionero, 2008).

El aprendizaje de los niños y niñas depende de las interrelaciones de éstos con las personas que forman su comunidad, entendida más allá de 
los profesores/as y de las propias familias. Desde este punto de vista, la participación de la comunidad es imprescindible para el correcto funcionamiento de las Comunidades de Aprendizaje (Capllonch \& Figueras, 2011; Elboj, et al., 2002; INCLUD-ED Consortium, 2009). Esta colaboración de la comunidad se concreta en la gestión global del centro, así como, en la participación regular o puntual de voluntarios/as y otros miembros de la comunidad que colaboran en distintos proyectos y con distintas funciones en el centro educativo. Este proceso colaborativo es bidireccional ya que no sólo sale beneficiado el centro escolar, los maestros y los niños y niñas, sino también todas las personas participantes. El proceso de participación comunitaria fortalece la propia comunidad ya que favorece el desarrollo de una ciudadanía activa, capaz de autogestionarse y de trabajar conjuntamente para mejorar su comunidad y la calidad de vida (Freire, 2012; García Yeste, Leena \& Petreñas, 2013).

Para comprender la aportación de la participación activa de los adultos no expertos en las Comunidades de Aprendizaje es imprescindible profundizar en dos conceptos básicos: la Zona de Desarrollo Próximoy la inteligencia cultural.

Vygotsky (1978) argumentaba que el aprendizaje humano se basa en nuestra naturaleza social, enfatizando claramente en la importancia de la colaboración e interacción en los procesos de aprendizaje. Así de forma coherente, el autor definió el concepto de Zona de Desarrollo Próximo como la distancia entre el desarrollo actual del niño o niña y su desarrollo potencial con la colaboración de un adulto o compañero más capaz. Para proseguir con nuestra argumentación, nos interesa especialmente entender que «Vygotsky no limita las interacciones con personas adultas a las realizadas con expertos, como el profesorado, sino que se refiere ampliamente a «guía adulta»» (Tellado \& Sava, 2010, p.163). El autor explicaba que las personas adultas no expertas proponían didácticas para presentar los contenidos mejorando los resultados de aprendizaje, pero que los aprendizajes no sólo se limitaban a aquellos considerados «académicos» o instrumentales sino que también se desarrollaban aprendizajes no académicos vinculados a distintas formas de aprender. Desde este punto de vista, la participación de las personas adultas de la comunidad, expertas y no expertas, tiene un papel fundamental en las prácticas educativas de éxito de las Comunidades de Aprendizaje.

Por otra parte, el concepto de inteligencia cultural, como elemento básico del aprendizaje dialógico que se desarrolla en otros artículos de este monográfico, nos ayuda también a fundamentar la importancia de la participación activa de los adultos en los centros escolares. Este concepto se basa en la superación de un concepto reduccionista de la inteligencia que la limita a una capacidad cognitiva y/o de habilidades prácticas. Ramis y Krastina (2010) explican que la inteligencia cultural «consists of academic intelligence (acquired in academic contexts), practical intelligence (acquired in daily contexts) and communicative intelligence. The basis of communicative intelligence is that all people are capable of speech and action» (p. 245). Desde este punto de vista, todas las personas adultas, expertas o no expertas, tienen una inteligencia cultural que les permite afrontar los retos de la vida. Estas inteligencias culturales tienen su espacio en los centros escolares pudiendo contribuir claramente al desarrollo educativo global de todos los niños y las niñas.

Hasta aquí hemos analizado la importancia de la participación de adultos expertos y no expertos de la comunidad en las Comunidades de Aprendizaje, así como argumentado las aportaciones de estos adultos a los procesos de enseñanza / aprendizaje partiendo de los conceptos de Zona de Desarrollo Próximo e Inteligencia Cultural. Así pues, entendemos que analizar el conflicto desde la perspectiva de los miembros adultos de las comunidades de aprendizaje obliga a ampliar la visión del «adulto» implicado. Es imprescindible tener en cuenta las valoraciones de los familiares de los niños y niñas, y voluntarios y voluntarias, que como miembros adultos de la comunidad educativa expertos o no expertos, se presentan como agentes educativos, al igual que el propio profesorado.

Si bien el conflicto puede surgir de forma global en todos los espacios escolares, las clases de educación física son posiblemente el espacio donde el conflicto se manifiesta de forma más presente y natural debido, entre otras razones, al carácter propiamente procedimental de sus contenidos que favorecen el contacto, la relación y la vivenciación compartida de sentimientos y actitudes. Así entendemos que la educación física, como área curricular, representa un espacio privilegiado para el tratamiento y prevención del conflicto escolar (Capllonch, 2008-2011).

Las formas que tienen los miembros adultos de la comunidad educativa (profesorado, familiares y voluntariado) de entender el conflicto, su tipología, sus especificidades, las razones por las que surgen, su gestión, las razones por las que se producen en las clases de educación física y, finalmente, las formas de resolución y prevención de los conflictos que se producen en las comunidades de aprendizaje, pueden ayudar a generar y potenciar mecanismos para gestionarlos desde el área de educación física.

\section{Objetivos}

El objetivo de este artículo se concreta en presentar las opiniones, creencias, y valoraciones que los miembros adultos (profesorado, familiares y voluntariado) de las Comunidades de Aprendizaje participantes en la investigación realizan sobre las causas de los conflictos que se producen en la educación física y las formas de abordarlos. De este objetivo se desprenden dos intenciones concretas u objetivos específicos. Por un lado identificar los elementos que dificultan la resolución comunitaria de los conflictos en las clases de educación física, y por otro, orientar las acciones que favorecen la resolución comunitaria de los conflictos en las clases de educación física, todo ello en los centros que son Comunidades de Aprendizaje.

\section{Método}

La metodología utilizada para la investigación es de orientación comunicativa-crítica. Esta metodología busca validar las explicaciones de los fenómenos e interacciones que se producen en la sociedad centrando especialmente la atención en aquellos elementos que generan exclusión y aquellos que pueden transformarla (Gómez, Latorre, Sánchez \& Flecha, 2006). En nuestro caso, la atención se fija en identificar aquellas situaciones que dificultan la aplicación de un modelo comunitario para la prevención y la resolución de los conflictos en la escuela y en las clases de educación física, y en cómo superar o transformar estas dificultades, a partir del estudio de la realidad desde la visión de los miembros adultos que conforman la comunidad educativa de estos centros.

La educación física ha sido el área curricular que ha servido como contexto en la investigación, porque su propia naturaleza la caracteriza como un medio eficaz e inclusor para que todos los niños y niñas puedan adquirir habilidades, conocimientos y valores (ICSSPE, 2010), a la vez que la hace idónea para el trabajo vinculado a la adquisición de competencias necesarias para gestionar el conflicto.

La muestra escogida para la investigación ha sido de 3 comunidades de aprendizaje en Cataluña y 3 comunidades de aprendizaje en Euskadi. En todas ellas se recogieron las aportaciones del profesorado, familiares y voluntariado a través de distintas técnicas de recogida de información, tanto cuantitativas como cualitativas.

En este artículo se presentan los resultados obtenidos mediante las técnicas de recogida de información cualitativa, y en concreto a través de entrevistas en profundidad a los miembros adultos de las comunidades de aprendizaje participantes en la investigación. Se realizaron un total de 36 entrevistas: 6 a profesores/as, uno de cada una de las comunidades de aprendizaje escogidas; 6 a familiares, uno de cada comunidad; y 6 a voluntarios/as, una persona de cada una de las comunidades de aprendizaje seleccionadas. A través del análisis cualitativo/comunicativo se estudian las dimensiones exclusoras y transformadoras respecto al conflicto, su prevención y resolución en la escuela y en las clases de educación física. En la presentación de los resultados se han seleccionado párrafos de las entrevistas que ilustran las valoraciones que hacen los diferentes miembros adultos de las comunidades de aprendizaje. En 
cada uno de los párrafos seleccionados aparecen las letras de P, V o F que hacen referencia a Profesor/a, Voluntario/a o Familiar respectivamente. A continuación aparecen las letras H o M para Hombre o Mujer, seguido del número de la entrevista y del párrafo seleccionado. Así, por ejemplo (PH3, 64) querría decir que se trata de la entrevista n. 3 realizada a un profesor hombre, y de la que se ha seleccionado el párrafo número 64.

\section{Resultados y discusión}

Barreras que impiden la resolución comunitaria de los conflictos Las barreras representan la dimensión exclusora, es decir, aquellas situaciones que generan conflictos e impiden la implementación del modelo comunitario de prevención y resolución de conflictos en la escuela y en concreto en la educación física.

\section{a) Desigual oportunidad de alcanzar el éxito}

Las clases de educación física basadas en modelos más tradicionales constituidos por juegos competitivos y eliminatorios, dificultan la integración y suelen generar conductoras de exclusión y marginación, enfrentamientos y conflictos (Blandón, Molina \& Vergara, 2005; Ortí, 2003). En efecto, cuando aparecen contenidos vinculados con la práctica deportiva, la baja tolerancia a la frustración que presentan algunos alumnos, la tendencia competitiva de los niños y las niñas a ganar, la excesiva búsqueda de la victoria por encima de la participación y las trampas para no perder en el juego, propician la generación de conflictos y dificultan su resolución a través del diálogo:

Pues no sé... pues ha sido gol, no ha sido gol o te has pasado de la línea o no te has pasado, me has empujado o me has quitado lo que es la pelota de las manos, no se puede... Entonces ya entre ellos empiezan a discutir. En algunos casos llegan a lo que es a las manos. (PH3, 64)

Otros aspectos que generan conflicto están vinculados al rechazo por el nivel de habilidad (Ortí, 2003) o la negativa a formar grupo con algunos niños y niñas en concreto, situaciones que alteran el ritmo y clima delaclase:

Bueno, sobre el conflicto... es de cuando uno gana o uno pierde y no se sabe aguantar que haya perdido. Conflictos de no quiero ir con este niño, conflictos de no me quiero llevar bien, no me aguanto, no quiero hacer educación física. Diferentes. Pero sobretodo en el juego, en el juego un niño hace trampas: conflicto. Un niño le haces un grupo que va con otro: no quiero ir con este, conflicto. Y así alterando el ritmo de la clase. (VH2, 42)

b) Influencia negativa de los medios de comunicación y falta de análisis crítico y acompañamiento de las familias

Algunos miembros adultos coinciden en que los medios de comunicación transmiten la idea de que la violencia es un sistema eficaz para la resolución de conflictos. La imagen de deportistas conocidos se repite continuamente, e incluso en ocasiones son sobrevaloradas y aplaudidas (Carlssonpaigne \& Levin, 1992). El alumnado más joven y más vulnerable reproduce estas acciones sin pensar que tienen una connotación negativa. Difícilmente desde la educación física se tratan estos temas o se critican estas actitudes:

Cuando surge un conflicto, la primera... la primera salida que tiene es la violencia, es empujarte, el pegarte, el atacar... y, efectivamente, los modelos que se están dando, vamos, ayudan muchísimo a reaccionar así, pero bueno una... la primera impresión es esa, la primera actuación es esa, yo pienso que sí, que los niños tienen... su primera reacción es violenta, cuando surge un conflicto, cuando algo les molesta, cuando algo no les ha gustado... en general, ¿¿eh?, no todos, pero sí que tienden a hacer una reacción violenta y sí que pienso que los juegos, la televisión, todo esto ayuda muchísimo a que esto sea así. (PM4, 129)

Si yo creo que cuando están viendo que unos personajes tan importantes como los jugadores de fútbol, te digo porque estos están todo el día jugando a futbol, se permiten dar patadas y hacen, porque hacen unas entradas tan fuertes, que luego ellos las imitan...entonces no les parece que esté mal porque lo están viendo todos los días y desde su casa mismo, desde la calle o desde los medios de comunicación, se está, no se está dando ningún tipo de importancia mala, han hecho unas entradas increíbles, uno se ha roto un pie pero no pasa nada, entonces para ellos no supone nada malo si lo están viendo todos los días, se supone que si los adultos lo hacen, no es tan malo. (PM1, 141-149)

En algunos casos los padres y madres opinan que el comportamiento del alumnado depende más de la educación familiar que de lo que puedan ver o escuchar en la televisión. En este sentido la falta de acompañamiento de los adultos cuando los niños y niñas se enfrentan a los medios de comunicación provoca la pérdida de criterios claros en el momento de interpretar las imágenes:

No lo sé, mira, yo creo que en cierta manera deben subir, pero también uno está ahí para controlar, o sea tú tienes que ver qué cosas ve tu hijo, y explicarle que lo que ve en la tele no es la realidad o es una ficción, a veces si es de una pelea que se disputan alguna cosa y se lo solucionan peleando, eso no quiere decir que eso tenga que ser en la vida real. Yo creo que en algunas cosas sí, y en otras o, pero como te digo tenemos que estar ahí pendientes de las cosas que ven nuestros hijos. (FM4, 39)

\section{c) Prevalencia del modelo autoritario de gestión del conflictos}

De los tres modelos de intervención para el tratamiento del conflicto (Aubert, et al., 2004), como son el modelo normativo o disciplinar, el modelo mediador o experto y el modelo comunitario, al menos la mitad de los adultos entrevistados admiten que en su centro escolar, el que predomina, es el primero. Se suele recurrir al castigo, y en función de la gravedad del conflicto, se aplican unas medidas u otras, que acostumbran a recogerse en el reglamento interno del centro.

En los resultados procedentes del análisis de las entrevistas en profundidad, el profesorado, familiares y voluntariado reconoce que en la escuela se usan métodos más bien asociados al modelo normativo o autoritario para la gestión de los conflictos, a saber: castigos, sanciones, o incluso expulsiones, dependiendo de la gravedad del conflicto, más que las resoluciones fundamentadas en el diálogo. Así pues, aunque existan evidencias de que en ocasiones se intente resolver los conflictos producidos en la escuela entre diversas partes de la comunidad educativa, hay aún momentos en que se toman decisiones desde el propio centro sin contar con otras opiniones. El propio centro establece unas normas, un protocolo a seguir, y los estudiantes y familiares que acuden al centro deben respetarlo:

Y bueno, cuando la criatura ya lleva tres partes, a no ser, se supone que han de ser tres partes, hay una expulsión. Que tú previamente ya has ido informado a las familias con los partes que te vuelven firmados. Sí que es cierto que a veces te saltas los tres partes, porque realmente se ha producido un conflicto muy grave, por una falta de respeto importantísima que no se puede permitir. Entonces pasas ya directamente al castigo inmediato. Avisas a las familias y se expulsa al crío. (PM5, 55)

Por otra parte, algunos miembros del profesorado entrevistado admiten que en ocasiones no utilizan la mediación ni el diálogo para resolver y prevenir los conflictos, sino una forma más autoritaria basada en amenazas y castigos, con objeto de agilizar el trámite y que el alumnado no provoque más conflictos por miedo a ser amonestado.

Entonces eso facilita pues que según qué tipo de conflictos se lo piensen un poco. Los pequeños igual no tanto porque es el calentón del momento pero sobretodo los mayores sí que se lo piensan dos veces, si pues no vas al viaje de fin de estudios ¡cuidado!, ¡cuidado! que me la estoy jugando mucho... si ellos lo saben y saben que consecuencia tiene su acción...entonces bueno hay algunos que son inevitables y pasan, pero otras sí que tienen consecuencias. (PM1, 196-202)

Se recoge entre los miembros adultos de la comunidad la dificultad que conlleva la implementación del modelo comunitario para la resolución y prevención de conflictos, en cuanto a tener que cambiar de 
costumbres, de maneras de actuar en la escuela y mayor disponibilidad delos familiares:

Porque la escuela está,... porque hay mucha vieja escuela y se hacen las cosas como se han hecho siempre y cuesta mucho de cambiar, en las escuelas... maneras de hacer. Quizá sería un poco las dos cosas, cambiar mentalidades y también gestionarlo, que también ponerlo en práctica, que toda la comunidad participe... difícil, pero que claro sería lo que se tendría que hacer. (VH3, 251257)

Lo que pasa es que eso, pide que haya una implicación por parte de los padres, o sea, de todos los participantes de la escuela, y a veces, no es tan fácil esa participación. Creo que como todo el mundo trabaja y tiene su vida y sus cosas pues, creo que es un poco más complicado que llevar el modelo disciplinar, por ejemplo. (VM4, 363-367)

\section{d) Dificultades de comunicación}

Otra idea que surge en las entrevistas se sitúa en la dificultad de llegar a acuerdos por la ausencia de competencias comunicativas que favorecerían el diálogo. Esta situación afecta tanto a profesorado como a familiares, voluntariado y alumnado. Amenudo suelen generarse discusiones, y la poca capacidad y recursos que tienen las personas para gestionar sus necesidades o dar salida a sus inquietudes provoca nuevos conflictos:

Se producen situaciones en las que tienen que ponerse a prueba las capacidades para negociar; por ejemplo el típico... ahora recuerdo que se producen más ocasiones para discutir, porque qué sé yo: tienen que discutir por quien se queda con la pelota, por quien ocupa este lugar o el otro... (VH1, 68)

Por otro lado, la escasa comunicación que, en algunos casos, se da entre familia y escuela, o la desigual participación entre padres y madres del alumnado, representa otro de los factores que dificultan el mantenimiento de modelos más normativos en la resolución de conflictos.

\section{e) Normalización del conflicto por parte de los adultos}

Una situación que consideramos grave es la normalización del conflicto que realizan los adultos. Éstos valoran que las riñas, las disputas, los insultos y las peleas forman parte del mundo infantil, que no suelen ser muy graves, que son habituales y de poca importancia. Esta forma de entender el conflicto favorece su permisividad e impide que se establezcan unas normas que permitan gestionarlo.

E: Los conflictos forman parte de la vida...

P:Sí, forman parte de la vida... se enfadan entre ellos, porque me ha empujado, porque no sé qué... ¿̇por qué te ha empujado? porque me ha hecho no sé qué, porque me ha tocado aquí, porque me ha tocado acá... Al final acaban enfadándose entre ellos, pero por tonterías de pues que iba corriendo y me ha empujado... y ¿̇por qué le empujas? ¡Ah, si no le he empujado!... sólo le he hecho así.. Son cosas de esas, jugando... porque me han tirado un balón y lo han tirado a propósito... son en el juego... (PM4, 91-92)

Por otro lado los familiares explican que los patrones de agresividad, racismo y otros malos comportamientos a menudo responden a conductas observadas y normalizadas en el hogar y al modelo que ejercen los miembros adultos próximos a los niños y niñas.

Mira yo creo que aquí al niño en cierta medida se le permiten muchas cosas. Hay padres que controlan muy poco a los niños. Te lo digo porque, a ver, yo he visto cosas aquí en el colegio que me han dejado estupefacta. (FM4, 25)

Lo que sí hemos detectado últimamente es que el racismo que los padres traen de casa, lo traen los niños, y lo hemos detectado sobre todo en los insultos. Enseguida salta el moro de mierda, el... cosas así, enseguida salta, enseguida. Porque bueno, es una cosa que traen de casa, yo lo tengo clarísimo, eso es algo que ya entra en el ámbito de la educación de casa. Entonces este tipo de insulto racista, o sea... aprovechas todas las armas que tienes para meterse con ese compañero. (FM5, 151-156)

\section{Estrategias que favorecen la resolución comunitaria de los con-} flictos

Representan la dimensión transformadora y muestran las formas para contribuir a la superación de los conflictos y de las barreras que impedirían la implementación de un modelo comunitario de prevención y resolución de conflictos en la escuela y en las clases de educación física, desde la perspectiva de los miembros adultos.

a) Consensuar con todos los miembros de la comunidad el funcionamiento y las normas para la prevención y resolución de conflictos que se producen en el área de educación física.

El profesorado entrevistado opina que es interesante que las familias se impliquen en la vida y decisiones del centro, para que toda la comunidad funcione conjuntamente y en la misma dirección. Para ello y en la línea argumentada por Aubert, Duque, Fisas y Valls (2004), es necesario propiciar situaciones en las que todos los miembros de la comunidad educativa dialoguen para llegar a acuerdos:

Es básico. O sea en la escuela hay dos palabras clave. Una es el trabajo cooperativo. La otra es el trabajo dialógico, el de hablar. Y por tanto estas dos cosas, en todos los ámbitos y todas las asignaturas deberían ser así. Por tanto, es que es básico. (PM2, 343)

En los centros que son comunidades de aprendizaje, los adultos valoran la creación de espacios en los que se facilita la posibilidad de encuentro entre profesorado, familiares y alumnado, bien mediante las asambleas de centro, de clase y/o grupos interactivos.

A ver, los conflictos normalmente se intentan resolver y hablarlo en las asambleas de clase, o sea, cada semana nosotros hacemos asamblea de clase. (PM2, 157)

Sí, sí todas las clases. Tenemos un espacio que es la asamblea de clase y otro que es el corro, que hacemos cada día. (PM2, 159)

Pues a través de delegados de clase por ejemplo, a través delegados de clase, reuniones con profesorado, transmitiendo las informaciones y habiendo consensuado previamente evidentemente las normas los pasos a seguir y teniendo muy claro el significado de lo que significa mediar, que no se lo tomen tampoco como un juego, es algo serio que va a repercutir en la vida, en la vida adulta. (PM5, 67)

Pues reuniendo, eso, a las partes, como se ha hecho, por grupos, asambleas... Mediante un cuestionario, donde se expresan los objetivos principales, y luego se van, de ahí, sacando las conclusiones entre todos, resumiendo las que, de todo el grupo, nos parecen más importantes y luego será, de todos los grupos, lo mismo, consensuar las que nos parezcan más importantes e ir clasificándoles por orden de prioridad y luego ya, para hacer un seguimiento, para ir revisándolas, por... ¿ ¿cómo se llama?, grupos de trabajo, por... comisiones de trabajo. Y así en eso estábamos, queriendo hacer eso. (PM6, 455-462)

Estos espacios deberían asegurar la creación de un sistema de normas específico para la educación física consensuado entre todos los miembros de la comunidad educativa: familiares, voluntarios/as, profesorado e incluso el alumnado tal como sugieren Flecha y García Yeste (2007).

Es la actitud que se toma frente a las familias, frente a los voluntarios, o sea la obertura, de que todo el mundo puede entrar, que todo el mundo tiene algo que decir, tú puedes aprender de todo el mundo y los otros de ti. (...) Esto es genial ¿̇no? es genial. Quiere decir que el hecho de ser tan participativa la escuela. (PM2, 273)

En ocasiones el profesorado prefiere que sea el propio alumnado el que, mediante el diálogo, gestione sus propios conflictos y los resuelvan entre ellos. A veces con ayuda de otros iguales o aportando soluciones entre todas y todos con objeto de contribuir al desarrollo de la ciudadanía activa, y con ello a la mejora de su comunidad y la calidad de vida (Freire, 2012; García Yeste, Leena \& Petreñas, 2013).

Pues nada intentar solucionar a ver qué ha pasado intentas averiguar qué es lo que ha pasado, intentas mediar que se pongan ellos de acuerdo para que ellos lo solucionen entre ellos (...) (PM1, 29-31) La idea es que un poco... las personas, pero que, por ejemplo, si ha pasado alguna cosa con algún niño de mi clase por ejemplo, los de 
la clase también se hacen partícipes de esto. Esto sí. En la asamblea puedes hablar ¿no? Ha pasado esto ¿vosotros como lo veis? Esto sí (PM2, 223)

Y se trata de que... dar espacio a las dos partes para hablar, porque a veces empiezan a hablar entre ellos, se atropellan, no se dejan, no escuchan y... bueno, y entre ellos, si es posible, buscar soluciones, saber ver si tiene razón, qué ha pasado o si se puede solucionar el problema. (PM4, 107)

b) Orientar el área de educación física hacia la adquisición de valores como el respeto, la tolerancia, la solidaridad y la convivencia a través de metodologías participativas y grupos interactivos.

El profesorado, voluntariado y familiares entrevistados también coinciden en la idea de que las actividades y juegos cooperativos son los que más ayudan a prevenir y a resolver conflictos de forma adecuada, ya que el alumnado trabaja en conjunto y coopera para lograr un objetivo común (Velázquez Callado, 2003, 2004). Además implican la interacción y el diálogo entre iguales, cosa que favorece la gestión del conflicto y la convivencia en el grupo.

Algunos adultos asimismo proponen organizar actividades en grupos interactivos (heterogéneos en todas sus dimensiones: edad, sexo, nivel de habilidad, etnia, etc.) tutorizados por un adulto donde el alumnado se beneficia de las acciones de los demás compañeros y compañeras y orienta las suyas propias para obtener objetivos comunes (Valls \& Kyriakides, 2013):

Sí. Si es que de hecho yo, ya te digo, yo creo que ya llevamos unos años oyendo hablar del juego cooperativo, y del trabajo cooperativo, que además, de hecho aquí en esta escuela se trabajan los grupos interactivos y los grupos interactivos es un trabajo cooperativo, entonces en EF, perfectamente, todavía es más fácil. Cualquier juego puedes adaptarlo, no, se puede volver no competitivo y sí cooperativo. (PM5, 94)

En este mismo sentido, el juego y las actividades multiculturales se valoran como una medida de proximidad que permitan conocerse mejor entre sí y desarrollar una actitud de empatía y solidaridad en el grupo (Gil Madrona \& Pastor Vicedo, 2003; Grimminger, 2011). Estos juegos contribuyen al enriquecimiento personal y a la cohesión del grupo, y se traduce en muchos casos en conocer y compartir juegos de su país de origen:

Sí, se puede hacer no sé la semana o el día de los juegos...xxx culturales, de diferentes culturas precisamente en esta escuela, bueno en ésta y en casi todas, no, debido al alto nivel de inmigración, hay un conjunto, bueno confluyen muchas culturas. Es una manera... podría ser, ya te digo, un día al mes, o una semana cultural no sólo de cara a la EF ¿eh?, dedicado a convivencia de diferentes culturas y venir familiares con sus juegos típicos, de sus países, juegos que además, si puede ser, que fueran cooperativos, se podría preparar. Un día o una semana de este tipo. (PM5, 102-104)

\section{c) Gestionar adecuadamente la competición}

Los adultos participantes verbalizan en las entrevistas en profundidad que las actividades competitivas no se consideran siempre exclusoras, ya que su potencial para la prevención y resolución de conflictos no depende de su carácter de competición, sino de cómo se enfoca, cómo se trata (Acuña Delgado \& Acuña Gómez, 2011). Una gestión adecuada de la competición orientada a la superación personal y al respeto del nivel de habilidad de los compañeros y compañeras contribuye a la prevención de los conflictos en las clases de educación física:

Yo pienso que depende de cómo es el enfoque ¿no? si se compite dentro de un mismo grupo... bueno, la idea es para mí que se realice algún tipo de actividad en la que competir signifique cooperar con los otros, donde la persona pueda inscribir de alguna manera su esfuerzo dentro de un esfuerzo colectivo o comunitario.(VH1, 78)

Y es que en efecto, la competición puede resultar valiosa en las clases de educación física si es justa para todos y todas y si se busca la superación personal (Velázquez Buendía, 2001). Una buena gestión de la competición implica dar las mismas oportunidades a todos los niños y niñas, independientemente de su nivel de habilidad y asegurar que todos y todas puedan aprender y conseguir el éxito.

Hombre, si la competición es como realización personal y como superación personal, yo sí que... a mí que una persona sea competitiva no me parece mal, pero que sea competitiva no a costa de los demás, o sea, de fastidiar a los demás, queél intente superarse como persona. (FM1, 339-342)

\section{Conclusiones}

Así pues, atendiendo a las evidencias recogidas a partir de la revisión teórica y a partir de los resultados obtenidos, podemos ofrecer algunas conclusiones, estructuradas en base a cuatro ejes principales, que exponemos a continuación:

El área de educación física (y las sesiones en las que se concreta) constituye, debido a sus características particulares, un espacio propicio para trabajar sobre la prevención y la resolución de conflictos.

En los centros que son comunidades de aprendizaje, en el contexto de la educación física también deberían quedar reflejados los principios de actuación que definen el modelo comunitario de prevención y resolución de conflictos: tanto por coherencia de funcionamiento, como para aprovechar las potencialidades que el área ofrece en este sentido.

Cabe destacar que podemos encontrar diversas barreras que dificultan la aplicación con éxito del modelo comunitario. Entre ellas, destacan: la posible falta de igualdad de oportunidades del alumnado para alcanzar el éxito en la asignatura de educación física; la influencia negativa de los medios de comunicación (especialmente si no se acompañan de análisis crítico en el entorno familiar); la prevalencia del modelo autoritario de resolución de conflictos; la posible falta de competencias comunicativas y recursos para el diálogo (presente tanto en profesores como en alumnado, familiares y voluntariado); y la normalización del conflicto por parte de los adultos.

Por eso, resulta importante (tal como se desprende de las aportaciones de los participantes) potenciar, dentro del área de educación física, aquellas estrategias que favorecen la aplicación del modelo comunitario; entre las que destacamos las siguientes: consensuar el funcionamiento del área, así como las normas para la prevención y resolución de los conflictos; orientar el área hacia la adquisición de valores (a través de metodologías que fomenten la participación); y utilizar adecuadamente la competición (que a menudo está vinculada a los contenidos del área) para poder aprovechar su potencialidad como espacio de vivencia de gestión del conflicto.

\section{Referencias}

Acuña Delgado, A., \& Acuña Gómez, E. (2011). Sport as platformfor values education. Journal of Human Sport and Exercice, 6(4), 574584.

Aubert, A., Duque, E., Fisas, M., \& Valls. E. (Eds). (2004). Dialogar y transformar. Pedagogía crítica del siglo XXI. Barcelona: Graó.

Aubert, A., Flecha, A., García Yeste, C., Flecha, R., \& Racionero, S. (2008). Aprendizaje dialógico en la Sociedad de la Información. Barcelona: Hipatia.

Blandón, M., Molina, V.A., \& Vergara, E. (2005). Los estilos directos y la violencia escolar. Las prácticas de la educación física. Revista Iberoamericana de Educación, 38, 87-103.

Capllonch, M. (2008-2011). Juega Dialoga y Resuelve. La superación de conflictos en educación física mediante el modelo comunitario. Diseño de un programa específico para comunidades de aprendizaje, con referencia, con referencia SEJ2007-61757/EDUC. Plan Nacional I+D+I.

Capllonch, M., \& Figueras, S. (2011). Educación física y comunidades de aprendizaje. Estudios Pedagógicos, 38(1), 231-247.

Carlssonpaigne, N., \& Levin, D.E. (1992). Making peace in violent times a constructivist approach to conflict-resolution. Young Children, 48(1), 4-13. 
Elboj, C., Puigdellívol, I., Soler, M., \& Valls, R. (2002). Comunidades de aprendizaje. Barcelona: Graó.

Flecha, R., \& García Yeste, C. (2007). Prevención de conflictos en las comunidades de aprendizaje. Idea La Mancha: Revista de Educación de Castilla La Mancha, 4, 72-76.

Freire, H. (2012). Una escuela abierta al barrio. Cuadernos de Pedagogía, 429, 53-57.

García Yeste, C., Leena, A., \& Petreñas, C. (2013, enero). Comunidades de aprendizaje. Scripta Nova, 17. Recuperado de: http:// www.ub.edu/geocrit/sn/sn-427/sn-427-7.htm\#cinco.

Gázquez, J.J., Pérez, M.C., \& Carrión. J.J. (2011). Clima escolar y resolución de conflictos según el alumnado: un estudio europeo. Revista de Psicodidáctica, 16(1), 39-58.

Gil Madrona, P., \& Pastor Vicedo, J.C. (2003). Actitudes multiculturales exteriorizadas en educación física: el estudio de un caso y la educación emocional como respuesta. Revista Complutense de Educación, 14(1), 133-158.

Grimminger, E. (2011). Intercultural competente among Sports and physical education teachers. Theoretical foundations and empirical verification. European Journal of Teacher Education, 3(34), 317331.

Gómez, J., Latorre, A., Sánchez, M., \& Flecha, R. (2006). Metodología Comunicativa Crítica. Barcelona: El Roure Ciencia.

INCLUD-ED Consortium. (2009). Acctions for success in schols in Europe. Bruselas: European Commission.

International Position Statement on Physical Education (2010). International Council of Sport Science and Physical Education
(ICSSPE). Recuperado de: http://www.icsspe.org/content/ international-position-statement-physical-education

Ortí, J. (2003). La resolución de conflictos en la educación física. Tándem. Didáctica de la Educación Física, 13, 40-50.

Ramis, M., \& Krastina, L. (2010). Cultural intelligence in school. Revista de psicodidáctica, 15(2), 239-252.

Tellado, I., \& Sava, S. (2010). The role of non-expert adult guidance in the dialogic construction of knowledge. Revista de psicodidáctica, 15(2), 163-176.

Valls, R., \& Kyriakides, L. (2013). The power of Interactive Groups: how diversity of adults volunteering in classroom groups can promote inclusion and success for children of vulnerable minority ethnic populations. Cambridge Journal of Education, 43(1), 1733.

Velázquez Buendía, R. (2001). Deporte: ¿presencia o negación curricular? In Actas del XIX Congreso Nacional de Educación Física (pp. 65-106). Murcia: Universidad de Murcia.

Velázquez Callado, C. (2003). El aprendizaje cooperativo en Educación Física: qué, para qué, por qué y cómo. En Ampliando horizontes a la cooperación: actas del III Congreso Estatal y IIberoamericano de Actividades Físicas Cooperativas. Valladolid: La Peonza.

Velázquez Callado, C. (2004). Las actividades físicas cooperativas. Una propuesta para la formación en valores a través de la educación física en las escuelas de educación básica. México: Secretaría deEducación Pública.

Vygotsky, L. (1978). Mind and society: the development of higher mental processes. Cambridge, MA: Harvard University Press.

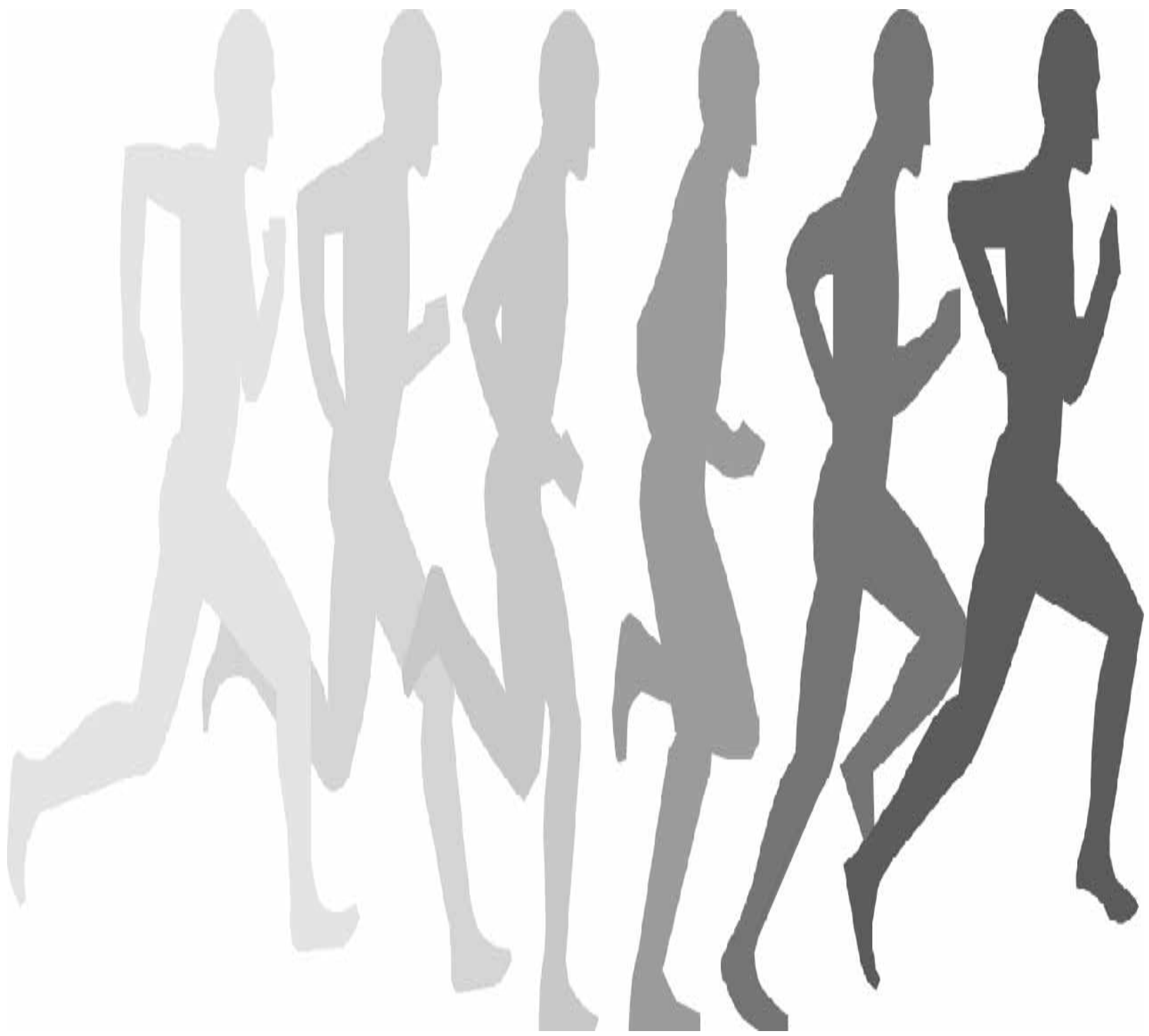

